\title{
SPECTRUM AND DIRECT INTEGRAL
}

BY

\author{
EDWARD A. AZOFF
}

ABSTRACT. Let $T=\int_{Z}^{\oplus} T(\xi)$ be a direct integral of Hilbert space operators, and equip the collection $\mathcal{C}$ of compact subsets of $C$ with the Hausdorff metric topology. Consider the [set-valued] function sp which associates with each $\varepsilon \in Z$ the spectrum of $T(\xi)$. The main theorem of this paper states that sp is measurable.

The relationship between $\sigma(T)$ and $\{\sigma(T(\varepsilon))\}$ is also examined, and the results applied to the hyperinvariant subspac? problem. In particular, it is proved that if $\sigma(T(E))$ consists entirely of point spectrum for each $\delta \in Z$, then either $T$ is a scalar multiple of the identity or $T$ has a hyperinvariant subspace; this generalizes a theorem due to $T$. Hoover.

1. Introduction. Let $T=\int_{Z}^{\oplus} T(\mathcal{E})$ be a direct integral. Roughly speaking (precise definitions will be given later), this means we are given a family $\{T(\xi)\}_{\mathcal{E} \in Z}$ of Hilbert space operators "depending measurably on the index $\tilde{E} "$ ". The main purpose of this paper is to examine the following two problems:

(1) How do $\{\sigma(T(\xi))\}$ depend on $\tilde{E}$ ?

(2) How does $\sigma(T)$ depend on $\{\sigma(T(E))\}$ ?

Intuitively, one feels the answer to the first question should be "measurably". In order to make this precise, we equip the collection $C$ of compact subsets of the plane with a certain natural Borel structure. This makes it meaningful to ask whether the [set-valued] correspondence $\mathcal{E} \rightarrow \sigma(T(\mathcal{E})$ ) is measurable. That such is the case constitutes the main result of $\$ 3$ (Theorem 3.5).

The second problem is taken up in $\$ 4$. Here, the results are somewhat discouraging; a simple example (4.4) shows that the spectrum of $T$ may have little relation to the spectra of $\{T(\mathscr{E})\}$. If one is willing to restrict $T$ to an appropriate reducing subspace however, some intelligent comments are possible. Our best result in this direction (Theorem 4.6) states that if each $T(E)$ has disconnected spectrum, then for a suitable $M, \sigma\left(\left.T\right|_{\mathfrak{M}}\right)$ will also be disconnected.

Received by the editors March 1, 1973 and, in revised form, November 12, 1973. AMS (MOS) subject classifications (1970). Primary 47A10, 47C15, 54C60; Secondary $47 \mathrm{~A} 15$.

Key words and phrases. Spectrum, direct integral, measurable set-valued function, finite topology, hyperinvariant subspace. 
The final section of the paper applies the above considerations to the hyperinvariant subspace problem. (A general discussion of the problem, along with references, can be found in $\$ 5$.) It is known that any operator with disconnected spectrum has a hyperinvariant subspace; applying Theorem 4.6, we see that if $\sigma(T(\mathcal{E}))$ is disconnected for each $\mathcal{E}$, then $T$ has a hyperinvariant subspace. A slightly more delicate argument (Theorem 5.10) yields a similar conclusion if $T$ is nonscalar and each $\sigma(T(\xi))$ is exclusively point spectrum. As a corollary, we obtain Hoover's theorem that every (nonscalar) n-normal operator has a hyperinvariant subspace.

In closing this introductory section, it seems appropriate to make two semiphilosophical remarks. First, although direct integral theory is usually thought of as a tool for studying operator algebras, it can also be helpful in investigating individual operators. Indeed, this approach is taken in Gilfeather's papers, [5] and [6], and is implicit in [9] and [13].

Finally, the tools used in this paper are all of a measure-theoretic nature. This seems natural in view of the fact that a direct integral is essentially a measure-theoretic entity. When the $\{T(E)\}$ act on a finite-dimensional space $H_{0}$, many measure-theoretic arguments can be replaced by continuity arguments; this is, in fact, the spirit of [9] and [13] (and [2] on which they depend). For insight into "why" such methods fail when $H_{0}$ is infinite-dimensional see [12].

2. Preliminary topics. In this section, we discuss briefly two concepts which will be fundamental to this paper: (1) the finite topology on $\mathcal{C}$, and (2) direct integral theory.

For a thorough discussion on methods of topologizing collections of subsets, see Michael [11]; we will follow [11] in matters of terminology and notation. Denote by $\mathcal{C}$, the collection of compact subsets of the complex plane. If $S_{1}, \cdots$, $S_{n}$ are subsets of $\mathbf{C}$, we write $\left\langle S_{1}, \cdots, S_{n}\right\rangle$ for $\left\{K \in \mathcal{C} \mid K \cap S_{i} \neq \varnothing, i=1, \cdots, n\right.$; $\left.K \subseteq \bigcup_{i=1}^{n} S_{i}\right\}$. Consider the family of subcollections, $\left\{\left\langle U_{1}, \cdots, U_{n}\right\rangle \mid n\right.$ is an integer; $\left\{U_{i}\right\}$ are open sets in $\left.C\right\}$. This forms a basis for a topology on $\mathcal{C}$ called the finite topology. (Although it will be of no concern to us here, it turns out that this topology coincides with the Hausdorff metric topology [8, \$28].)

In the sequel, we will always regard $\mathcal{C}$ as equipped with the finite topology and with the Borel structure subordinate to this topology. It thus becomes meaningful to speak of a map between some measure space $Z$ and $\mathcal{C}$ being measurable. At several points, we will make use of the existence of a measurable choice function for $\mathcal{C}$, i.e., a function $\phi_{0}: \mathcal{C} \rightarrow \mathrm{C}$ which is measurable (relative to the Borel structures on $\mathcal{C}$ and $\mathrm{C}$ ) and satisfies $\phi_{0}(K) \in K$ for each compact $K$. An existence proof can be found in Corollary 2 of [10]. 
We now consider a few of the rudiments of direct integral theory. Our discussion (and terminology) will be based on Dixmier [3], especially the first two sections of Chapter II.

Let $\mathcal{H}_{0}$ be a fixed separable Hilbert space and $(Z, \nu)$ a fixed standard measure space [3, p. 140]. We allow $\mathcal{H}_{0}$ to be finite dimensional. For each $\mathcal{E} \in Z$, set $\mathcal{H}(\xi)=\mathcal{H}_{0}$. We then form $\mathcal{H} \equiv \int_{Z}^{\oplus} \mathcal{H}(\xi)$, the direct integral (corresponding to the collection of constant vector fields) of the Hilbert spaces $\mathcal{H}(\mathscr{E})$. This consists by definition, of all [equivalence classes of] functions $x(\cdot): Z \rightarrow H_{0}$ satisfying

(1) for each $y \in \mathcal{H}_{0}$ the scalar valued function $\mathscr{E} \rightarrow(x(\xi), y)$ is measurable, and

(2) $\int_{Z}\|x(\xi)\|^{2} d \nu<\infty$.

One defines an inner product on $\mathcal{H}$ by setting $(x(\cdot), y(\cdot)) \equiv \int_{Z}(x(\mathcal{E}), y(\mathcal{E})) d \mathcal{E}$; this makes $\mathcal{H}$ into a (complete, separable) Hilbert space.

Suppose for each $\mathcal{E} \in Z$, we have an operator $T(\mathcal{E})$ on $\mathcal{H}(\mathcal{E})$ such that

(1) the function: $\mathcal{E} \rightarrow(T(\mathcal{E}), x, y)$ is measurable for each pair of vectors $x$ and $y$ in $\mathcal{H}_{0}$, and

(2) ess sup $\|T(\xi)\|$ is finite.

We then define $\int_{Z}^{\oplus} T(\mathscr{f})$, the direct integral of $\{T(\mathscr{E})\}$ by the formula:

$$
\int_{Z}^{\oplus} T(\mathcal{E})(x)(\Re)=T(\Re) x(\Re), \quad x \in \mathcal{H}, \Re \in Z .
$$

This is a bounded operator on $\mathcal{H}$ with norm equal to ess sup $\|T(\mathcal{E})\|$. It should be noted that, in the case when $Z$ is discrete, the concept of direct integral reduces to that of direct sum.

In the sequel, $T=\int_{Z}^{\oplus} T(\mathcal{E})$ will denote a fixed direct integral operator on $\mathcal{H}$. Following Dixmier, we will say an operator in $\mathfrak{L}(\mathcal{H})$ is decomposable if it can be expressed as a direct integral.

3. Measurability of the spectral function. The objects $Z, H_{0}, \mathcal{H}$, and $T$ constructed above are to be regarded as fixed. We define the spectral function of $T$ (denoted $\mathrm{sp}_{T}$ or $\mathrm{sp}$ ) by the correspondence $\mathcal{E} \rightarrow \sigma(T(\mathcal{E})$ ). The main purpose of this section is to show that $\mathrm{sp}$ is measurable (as a function between $Z$ and $\mathcal{C}$ ). This is accomplished in Theorem 3.5 through the aid of several introductory lemmas.

Lemma 3.1. Let $\mathcal{K}$ be a separable Hilbert space and $A \in \mathscr{L}(\mathcal{K})$. Choose $\left\{y_{k}\right\}_{k=1}^{\infty}$ to be a countable dense subset of the unit spbere $(\{y \mid\|y\|=1\})$ of $\mathcal{K}$. Then the following are equivalent:

(1) $A$ is invertible and $\left\|A^{-1}\right\| \leq n$. 
(2) $\inf _{k}\left\|A y_{k}\right\| \geq 1 / n$ and $\inf _{k}\left\|A^{*} y_{k}\right\| \geq 1 / n$.

Proof. (1) $\Rightarrow(2)$. Immediate.

$(2) \Rightarrow(1)$. Use the open mapping theorem.

Lemma 3.2. Let $A$ and $\left\{y_{k}\right\}_{k=1}^{\infty}$ be as above. Suppose moreover that $K$ is a compact subset of $\mathbf{C}$ and $\left\{\lambda_{n}\right\}_{n=1}^{\infty}$ is a dense subset of $K$. Then the following are equivalent:

(1) The spectrum of $A$ is disjoint from $K$.

(2) $\inf _{k, n}\left\|\left(A-\lambda_{n} I\right) y_{k}\right\|>0$ and $\inf _{k, n}\left\|\left(A-\lambda_{n} I\right)^{*} y_{k}\right\|>0$.

Proof. (2) $\Rightarrow(1)$. Suppose (2) holds. Then for each $\lambda \in K, \inf _{k}\left\|(A-\lambda) y_{k}\right\|$ and $\inf _{k}\left\|(A-\lambda I)^{*} y_{k}\right\|$ are both nonzero and, hence, $(A-\lambda I)$ is invertible by Lemma 3.1.

$(1) \Rightarrow$ (2). Suppose (2) fails. (For definiteness, say $\inf _{n, k}\left\|\left(A-\lambda_{n} I\right) y_{k}\right\|=0$.) We get a sequence of integers $\left\{n_{j}\right\}_{j=1}^{\infty}$ such that $\inf _{k}\left\|\left(A-\lambda_{n_{j}} I\right) y_{k}\right\| \stackrel{j}{\rightarrow} 0$. Dropping down to a subsequence, we may assume $\lambda_{n_{j}} \rightarrow \lambda_{0}$. Hence $\inf _{k}\left\|\left(A-\lambda_{0} I\right) y_{k}\right\|$ $=0$ and $\left(A-\lambda_{0} l\right)$ is not invertible. Thus (1) fails.

Remark 3.3. For future use, note that the proof of Lemma 3.2 actually shows that $\sup _{\lambda \in K}\left\|(A-\lambda I)^{-1}\right\|=\left[\inf _{k, n}\left\|\left(A-\lambda_{n} I\right) y_{k}\right\|\right]^{-1}$.

Lemma 3.4. The Borel structure on $\mathcal{C}$ is generated (as a $\sigma$-algebra) by the family

* $\quad\{\langle V\rangle \subseteq \mathcal{C} \mid$ the complement of $V$ is a compact subset of $\mathrm{C}\}$

of subcollections of $\mathcal{C}$.

Proof. By definition, the Borel structure $B$ on $\mathcal{C}$ is generated by the family

$$
\left\{\left\langle V_{1}, \cdots, V_{n}\right\rangle \subseteq \mathcal{C} \mid V_{1}, \cdots, V_{n} \text { are open }\right\}
$$

But $\left\langle V_{1}, \ldots, V_{n}\right\rangle=\left\langle\bigcup_{i} V_{i}\right\rangle \backslash \bigcup_{j}\left\langle\bigcup_{i \neq j} V_{i}\right\rangle$ so $\mathfrak{B}$ is generated by the family

$$
\{\langle V\rangle \subseteq \mathcal{C} \mid V \text { open }\}
$$

Now any open subset $V$ of the complex plane can be expressed as the countable intersection of sets $\left\{V_{n}\right\}_{n=1}^{\infty}$ with compact complement. Since $\langle V\rangle=\bigcap_{n=1}^{\infty}\left\langle V_{n}\right\rangle$, we see that the family * generates $\mathfrak{B}$, as desired.

Theorem 3.5. sp is measurable.

Proof. Recall that we have $T=\int_{Z}^{\oplus} T(\mathscr{E})$ and sp: $Z \rightarrow \mathcal{C}$ by the correspondence $\xi \rightarrow \sigma(T(\xi))$. Let $K$ be a fixed compact subset of the plane and choose sequences 
$\left\{\lambda_{n}\right\}$ and $\left\{y_{k}\right\}$ dense in $K$ and the unit sphere of $\mathcal{H}_{0}$ respectively. Then, by virtue of Lemma 3.2 we have $\mathcal{E} \in \mathrm{sp}^{-1}\left(\left\langle K^{c}\right\rangle\right)$ if and only if both $\inf _{k, n}\left\|\left(A(\xi)-\lambda_{n} l\right) y_{k}\right\|$ and $\inf _{k, n}\left\|\left(A(\xi)-\lambda_{n} l\right)^{*} y_{k}\right\|$ are positive. Thus $\operatorname{sp}^{-1}\left(\left\langle K^{c}\right\rangle\right)$ is measurable for each compact $K$ and the proof is complete by Lemma 3.3.

Let $f: Z \rightarrow \mathcal{C}$. When is $f$ the spectral function of some decomposable operator $T$ ? One necessary condition (Theorem 3.5) is that $f$ be measurable. Borrowing a result from $\$ 4$ (Theorem 4.3 ) we see that $f$ must also be essentially bounded (i.e., there exists a fixed compact set $K$ of $\mathbf{C}$ such that $f(\mathcal{E}) \subseteq K$ for almost all $\mathscr{E}$ ). To conclude this section, we will show these two conditions are also sufficient.

Lemma 3.6. There exists a countable collection $\left\{\phi_{n}\right\}_{n=1}^{\infty}$ of measurable choice functions for $\mathcal{C}$ such that for each $K \in \mathcal{C}$, the set of points $\left\{\phi_{n}(K)\right\}_{n=1}^{\infty}$ is dense in $K$.

Proof. Let $\phi_{0}$ be a fixed measurable choice function on $\mathcal{C}$. For $\lambda \in \mathbf{C}$, and $\epsilon>0$, denote by $B_{\epsilon}(\lambda),\{z \in \mathbf{C}|| z-\lambda \mid \leq \epsilon\}$. Define $\phi_{\lambda \epsilon}: \mathcal{C} \rightarrow \mathbf{C}$ by

$$
\phi_{\lambda \epsilon}(K)= \begin{cases}\phi_{0}\left(K \cap B_{\epsilon}(\lambda)\right) & \text { if } K \cap B_{\epsilon}(\lambda) \neq \varnothing, \\ \phi_{0}(K) & \text { otherwise. }\end{cases}
$$

Note that each $\phi_{\lambda \epsilon}$ is measurable. The collection, $\left\{\phi_{\lambda \epsilon} \mid \lambda\right.$ has rational coordinates, $\epsilon$ is rational $\}$ then satisfies the lemma.

Theorem 3.7. Let $f: Z \rightarrow \mathcal{C}$. The following are equivalent:

(1) $f$ is measurable and essentially bounded.

(2) $f=\mathrm{sp}_{T}$ for some decomposable $T$.

Proof. We need only show (1) $\Rightarrow(2)$. Thus assume $f$ satisfies (1). Let $\mathcal{H}_{0}$ be an (infinite-dimensional) Hilbert space with orthonormal basis $\left\{e_{n}\right\}_{n=1}^{\infty}$ and choose $\left\{\phi_{n}\right\}_{n=1}^{\infty}$ as in Lemma 3.6. Define $T(\mathcal{G}) \in \mathscr{Q}\left(\mathcal{H}_{0}\right)$ to be the operator corresponding to the matrix $\operatorname{diag}\left(\phi_{n}(f(\mathcal{E}))\right)$. Clearly, $f(\varepsilon)$ is the spectrum of $T(\xi)$ and hence $f=\mathrm{sp}_{T}$ for $T=\int_{Z}^{\oplus} T(\xi)$.

4. The spectrum of $T$ versus the spectra of $\{T(\xi)\}$. In this section, we try to answer the following question: Knowing the spectra of $\{T(\mathcal{E})\}$, what can one say about the spectrum of $T=\int_{Z}^{\oplus} T(\mathcal{E})$ ? Unfortunately, the answer is "not much" (Example 4.4), though we do obtain a partial result in Theorem 4.3. Rather than give up the problem entirely, we change the question slightly: What can one say about the restrictions of $T$ to reducing subspaces? Our best result in this direction is Theorem 4.6. The proof of the following lemma is taken from Chow [1]. 
Lemma 4.1. Let $T=\int_{Z}^{\oplus} T(\mathcal{E})$. Then the following are equivalent:

(1) $T$ is invertible and $\left\|T^{-1}\right\| \leq n$.

(2) $T(\xi)$ is invertible for almost all $\mathcal{E}$ and ess sup $\left\|T(\mathcal{E})^{-1}\right\| \leq n$.

Proof. $(2) \Rightarrow(1)$. The hypothesis implies that both $T$ and $T^{*}$ are bounded below by $1 / n$. Hence (1).

$(1) \Rightarrow(2)$. The decomposable operators form a von Neumann subalgebra of $\mathcal{L}(\mathcal{H})$. Hence $T^{-1}$ must be decomposable. Say $T^{-1}=\int_{Z}^{\oplus}(\mathcal{E})$. Then for almost all $\mathscr{E}, S(\mathscr{E}) T(\mathcal{G})=T(\mathcal{G}) S(\mathcal{G})=I$. Thus $T(\mathcal{G})$ is invertible for almost all $\mathcal{E}$ and ess sup $\|(T(\xi))-1\|=$ ess sup $\|S(\xi)\|=\left\|T^{-1}\right\|$.

Example 4.2. It is an immediate consequence of Lemma 4.1 that if

$*$

$\{\tilde{E} \in Z \mid \lambda \in \sigma(T(\mathcal{G}))\}$ has positive measure,

then $\lambda \epsilon \sigma(T)$. The weakness of this assertion is demonstrated by considering multiplication by the independent variable on $L_{2}[0,1]$. (Here $\mathcal{H}_{0}$ is one dimensional, $Z=[0,1]$ and $T(\tilde{E})=\varepsilon I$.)

In this case, no $\lambda$ satisfies $*$ but $\sigma(T)=[0,1]$. We can do a bit better with the aid of the following theorem.

Theorem 4.3. Suppose $\sigma(T)$ is disjoint from the compact set $K$. Then for almost all $\mathcal{E}, \sigma(T(\mathcal{E})$ ) is disjoint from $K$.

Proof. Suppose $\sigma(T) \cap K=\varnothing$ and choose $\left\{\lambda_{n}\right\}_{n=1}^{\infty}$ dense in $K$. Then $\sup _{n}\left\|\left(T-\lambda_{n} I\right)^{-1}\right\|$ is finite; call this number $s$.

Now for each integer $n$ there is a set $E_{n}$ of measure zero such that $\sup _{\mathcal{E} E E_{n}}\left\|\left(T(\tilde{\xi})-\lambda_{n} l\right)^{-1}\right\| \leq s$ (Lemma 4.1). Set $E=\bigcup E_{n}$. Then $\left.\sup _{E \in E} \sup _{n} \| T(\mathcal{G})-\lambda_{n} l\right)^{-1} \| \leq s$. Note that for any operator $A$ and any $\lambda$,

$$
\left\|(A-\lambda I)^{-1}\right\| \geq 1 / \operatorname{dist}(\lambda, \sigma(A)) .
$$

It follows that for $\mathcal{E} \notin E,(T(\mathcal{E})-\lambda I)$ is invertible for each $\lambda \in K$.

Suppose each closed ball about the complex number $\lambda$ intersects $\sigma(T(E))$ for $\mathcal{E}$ in a set of positive measure. Since the spectrum of $T$ is closed, the last theorem shows $\lambda \in \sigma(T)$. Let $\hat{\sigma}(T)$ be the set of all $\lambda$ satisfying this condition. We call $\hat{\sigma}(T)$ the synthetic spectrum of $T$. Our theorem shows $\hat{\sigma}(T) \subseteq \sigma(T)$; for the $T$ of Example 4.2, $\hat{\sigma}(T)=\sigma(T)=[0,1]$.

Example 4.4. Take $Z=N$ with the counting measure; $\mathcal{H}_{0}=l_{2}(\mathrm{~N})$. For each $n \in \mathrm{N}$, set $T(n)$ to be the weighted shift with weights $(1,1, \ldots, 1,0, \ldots)(1$ occurs $n$ times). Then $\hat{\sigma}(T)=\{0\}$ but $\sigma(T)$ is the closed unit disc. (This is a slight variation of Solution 81 in [7].)

At this point, one might well give up the problem, but we will make one more 
attempt. If $E \subseteq Z$ is measurable, we can form $\mathcal{H}_{E}=\int_{E}^{\oplus} \mathcal{H}(\tilde{E})$ and $T_{E}=\int_{E}^{\oplus} T(\xi)$. Then $\mathcal{H}_{E}$ reduces $T$ and $T_{E}$ is the restriction of $T$ to $\mathcal{H}_{E}$. In the next two theorems, we examine the spectra of $\left\{T_{E}\right\}$.

Remark. The results of this section are closely related to Lemma 2.1 of [1]. In particular, it is not difficult to see that $\hat{\sigma}(T)=\bigcap\left(\bigcup_{\delta \in \delta}(T(\xi)) \mid \delta\right.$ is a set of full measure $\}$, and the proof of Theorem 4.3 does not differ appreciably from Chow's arguments. On the other hand, the measurability considerations of the preceding section play a crucial role in the following two proofs.

Theorem 4.5. Let $U$ be an open set containing $\hat{\sigma}(T)$. Then for some $E$ (positive measure) $\sigma\left(T_{E}\right) \subseteq U$.

Proof. For each $\lambda \in U^{C}$, there is an open set $\mathcal{O}_{\lambda} \ni \lambda$ such that for almost all $\mathcal{E}, \sigma(T(\mathcal{E}))$ is disjoint from $\mathcal{O}_{\lambda} \cdot U^{C}$ is covered by countably many of the $\left\{\mathcal{O}_{\lambda}\right\}$. Thus for almost all $\tilde{E}, \sigma(T(\mathcal{E}))$ is disjoint from $U^{C}$.

Set $f(\mathcal{E})=\sup \left\{\|T(\mathcal{E})-\lambda I\|^{-1} \mid \lambda \in U^{C}\right\}$. Applying the continuity of the resolvent, we see $f$ is finite almost everywhere. Also (Remark 3.3) $f$ is measurable. Thus for an appropriate integer $N, f^{-1}([0, N])$ has positive measure. Set $E=$ $f^{-1}([0, N])$. It follows from Lemma 4.1, that $\sigma\left(T_{E}\right)$ is disjoint from $U^{C}$.

Of course it may happen that $\sigma\left(T_{E}\right)$ is much smaller than $\hat{\sigma}(T)$. Nevertheless, we have the following.

Theorem 4.6. Suppose $\sigma(T(\mathcal{E}))$ is disconnected for each $\mathcal{E}$. Then for some $E$ (positive measure), $\sigma\left(T_{E}\right)$ is also disconnected.

Proof. Let $\mathcal{C}_{1} \subseteq \mathcal{C}$ be the collection of all (closed) squares in the complex plane whose corners have rational coordinates, and set $\mathcal{C}_{2}=$ \{finite unions of sets in $\left.\mathcal{C}_{1}\right\}$. Now, for each $\mathcal{E} \in Z$, there exist disjoint sets $K_{1}$ and $K_{2}$ in $\mathcal{C}_{2}$ such that $\operatorname{sp}(\mathscr{E}) \in\left\langle K_{1}, K_{2}\right\rangle$. Also sp is measurable and the collection $\mathcal{C}_{2}$ is countable. Hence we can find a set $F \subseteq Z$ of positive measure and fixed sets $K_{1}$ ' $K_{2} \in \mathcal{C}_{2}$ such that $\operatorname{sp}(\mathcal{E}) \in\left\langle K_{1}, K_{2}\right\rangle$ for each $\mathcal{E} \in F$.

Let $V_{1}$ and $V_{2}$ be disjoint open sets containing $K_{1}$ and $K_{2}$ respectively. Applying Theorem 4.5 to $T_{F}$, we find a subset $E$ of $F(\nu(E)>0)$ such that $\sigma\left(T_{E}\right) \subseteq V_{1} \cup V_{2}$. Moreover (Theorem 4.3), $\sigma\left(T_{E}\right)$ intersects both $K_{1}$ and $K_{2}$. Thus $T_{E}$ has disconnected spectrum.

5. An application to hyperinvariant subspaces. Let $A$ be a (bounded, linear) operator on a Hilbert space $\mathcal{K}$ and $\mathbb{N}$ a nontrivial (closed) subspace of $\mathcal{K}$ invariant under each operator commuting with $A$. Then $M$ is said to be byperinvariant for $A$. It is immediate that no scalar multiple of $I$ can have a hyperinvariant subspace. Whether every other operator has one is open; an affirmative answer 
would solve the invariant subspace problem. For a general summary of the work done on the hyperinvariant subspace problem, see [4].

In this section, we will examine the relationship between direct integrals and hyperinvariant subspaces. The special case of direct sums (i.e., $Z$ is discrete) can be handled using the concept of disjoint pair; the situation is completely described by Theorem 5.5. In the general case, we cannot do quite as well. Our main results are Theorems 5.9 and 5.10 .

A pair of operators $A_{1} \in \mathscr{L}\left(\mathcal{H}_{1}\right)$ and $A_{2} \in \mathscr{L}\left(\mathcal{H}_{2}\right)$ is said to be disjoint if 0 is the only bounded operator in $\mathcal{L}\left(\mathcal{H}_{2}, \mathcal{H}_{1}\right)$ satisfying $A_{1} X=X A_{2}$; this concept, implicit in [13] and formalized in [4], will play a crucial role in Theorem 5.5 below. Proposition 5.1 summarizes several well-known techniques for constructing hyperinvariant subspaces.

Proposition 5.1. Let $A$ (nonscalar) $\in \mathscr{L}(\mathcal{K})$ and suppose $\mathbb{M}$ and $\pi$ are nontrivial subspaces of $\mathcal{K}$. Eacb of the following conditions is sufficient to guarantee that $A$ bave a byperinvariant subspace:

(1) A bas nonempty point spectrum.

(2) $A^{*}$ bas a byperinvariant subspace.

(3) A does not have dense range.

(4) $M$ is invariant under $A, \pi$ is invariant under $A^{*}$, and the pair $\left(P_{\pi} A_{\pi}, A_{\pi}\right)$ is disjoint.

(5) $M$ is invariant under $A, \pi$ reduces $A$, and the spectra of $A_{\pi}$ and $A_{\pi}$ are disjoint.

(6) A bas disconnected spectrum.

Proof. (1) Any eigenspace of $A$ is hyperinvariant for $A$.

(2) If $\mathbb{M}$ is hyperinvariant for $A^{*}$, then $\mathbb{M}^{\perp}$ is hyperinvariant for $A$.

(3) If $A$ does not have dense range, then zero is an eigenvalue for $A^{*}$.

(4) See Theorem 2.5 of [4].

(5) If $A_{\pi}, A_{\Re}$ have disjoint spectra then both pairs $\left(A_{\pi}, A_{\Re}\right)$ and $\left(A_{\Re}, A_{\pi}\right)$ are disjoint. (Second paragraph on p. 302 of [13].)

(6) Let $\rho$ be a nontrivial spectral set of $A$. Then $E(\rho)$, the spectral projection associated with $\rho$, commutes with every operator commuting with $A$. Since $E \neq 0$ or $l$, either the null space of $E$ or the closure of the range of $E$ is nontrivial, and hence hyperinvariant for $A$.

The next lemma is a standard obvious reformulation of the hyperinvariant subspace problem. For $x \in \mathcal{K}$ and $A \in \mathfrak{L}(\mathcal{K})$, we write $A^{\prime}$ for the commutant of $A$ and $x_{x}^{A^{\prime}}$ for the closure of $\left\{B x \mid B \in A^{\prime}\right\}$; this notation conforms with Dixmier.

Lemma 5.2. In order for $A \in \mathfrak{L}(\mathcal{K})$ to bave a byperinvariant subspace, it is necessary and sufficient that for some nonzero $x \in \mathcal{K}$, the subspace $\mathscr{X}_{x}^{A^{\prime}}$ not be equal to K. 
Proof. $(-) X_{x}^{A^{\prime}}$ is invariant under $A^{\prime}$.

$\Rightarrow$ S Suppose $\mathbb{N}$ is hyperinvariant for $A$ and choose $x \neq 0$ in $\pi$. Then $M$ contains $X_{x}^{A^{\prime}}$ but $\mathbb{M}$ is proper.

Proposition 5.3. Let $A_{1} \in \mathfrak{L}\left(\mathcal{K}_{1}\right)$ and $A_{2} \in \mathfrak{L}\left(\mathcal{K}_{2}\right)$. Then $A_{1} \oplus A_{2}$ bas a byperinvariant subspace if and only if either

(1) $A_{1}$ bas a byperinvariant subspace, or

(2) $A_{2}$ bas a byperinvariant subspace, or

(3) the pair $\left(A_{1}, A_{2}\right)$ is disjoint, or

(4) the pair $\left(A_{2}, A_{1}\right)$ is disjoint.

Proof. Every operator $B$ in $\mathcal{L}\left(\mathcal{K}_{1} \oplus \mathcal{K}_{2}\right)$ can be represented by a $2 \times 2$ (operator) matrix

$$
\left[\begin{array}{ll}
B_{11} & B_{12} \\
B_{21} & B_{22}
\end{array}\right] \text { where } B_{i j} \in \mathscr{L}\left(\mathcal{K}_{j}, \mathcal{K}_{i}\right)
$$

Note that in order for $B$ to commute with $A_{1} \oplus A_{2}$, it is necessary and sufficient that $A_{i} B_{i j}=B_{i j} A_{j}$ for $i, j=1,2$.

Sufficiency of (1). Assume $A_{1}$ has a hyperinvariant subspace. Pick $x \neq 0$ in $\mathcal{K}_{1}$ such that $X_{x}^{A} \neq \mathcal{K}_{1}$. By the above comments about matrices, it follows that $\mathscr{X}_{x \oplus 0}^{A^{\prime}} \neq \mathcal{K}_{1} \oplus \mathcal{K}_{2}$.

Sufficiency of (3). Suppose $\left(A_{1}, A_{2}\right)$ is disjoint and choose $x \neq 0$ in $\mathcal{H}_{2}$. Then every vector in $X_{0 \oplus x}^{A^{\prime}}$ has 0 in its first coordinate. Thus $X_{0 \oplus x}^{A^{\prime}} \neq K_{1} \oplus K_{2}$.

Sufficiency of (2) and (4). Similar.

Necessity. Let $x_{1} \oplus x_{2}$ be a nonzero vector in $\mathcal{K}_{1} \oplus \mathcal{K}_{2}$. Without loss of generality, we assume $x_{1} \neq 0$. Now $\left[\begin{array}{cc}B_{11} & 0 \\ 0 & 0\end{array}\right]$ commutes with $A_{1} \oplus A_{2}$ for each $B_{11}$ commuting with $A_{1}$. By assumption $A_{1}$ has no hyperinvariant subspaces so $X_{x_{1} \oplus x_{2}}^{A^{\prime}}$ contains $\mathcal{K}_{1} \oplus 0$.

Choose $B_{21} \neq 0$ such that $A_{2} B_{21}=B_{21} A_{1}$. Then $\left[\begin{array}{ll}0 & 0 \\ B_{21} & 0\end{array}\right]$ commutes with $A_{1} \oplus A_{2}$ and so $X_{x_{1} \oplus x_{2}}^{A^{\prime}}$ contains nonzero vectors in $0 \oplus \mathcal{K}_{2}$. The argument of the preceding paragraph shows $\mathscr{X}_{x_{1} \oplus x_{2}}^{A^{\prime}}$ also contains all of $0 \oplus K_{2}$ and hence equals $K_{1} \oplus K_{2}$.

Corollary 5.4. Suppose $T_{E}$ bas a byperinvariant subspace. Then so does $T$.

Proof. $T=T_{E} \oplus T_{Z \backslash E}$

Theorem 5.5. Suppose $Z$ is discrete and no atom in $Z$ bas measure zero. Let $T=\int_{\mathcal{L}}^{\oplus} T(\mathcal{E})$ be a decomposable operator in $\mathcal{L}(\mathcal{H})$. Then $T$ bas a byperinvariant subspace if and only if either: 
(1) some $T(\mathcal{E})$ bas a byperinvariant subspace, or

(2) some pair $(T(\mathcal{E}), T(\Re))$ is disjoint.

Proof. The proof is a slight (but messy) modification of the proof of Proposition 5.3, and hence is omitted.

What is the proper analogue of Theorem 5.5 when $Z$ is not discrete? Certainly condition (1) makes no sense in this case, for $\{T(\mathcal{E})\}$ are only determined up to a set of measure zero. A reasonable sufficient condition is provided by the following conjecture.

Conjecture 5.6. Suppose $T(\mathcal{E})$ bas a byperinvariant subspace for eacb $\mathscr{E} \in Z$. Then $T$ bas a byperinvariant subspace.

As a "proof" of this statement, one might be tempted to form $\mathbb{M}=\int_{Z}^{\oplus \Uparrow \pi(\mathcal{G})}$ where $\Uparrow(\mathcal{E})$ is hyperinvariant for $T(\mathcal{E})$. Even leaving aside the problem of choosing $\{\pi(\xi)\}$ measurably, this approach fails since the commutant of $T$ may contain nondecomposable operators. (We avoided this problem in the discrete case by choosing all the $\{\pi(\xi)\}$ except one to be zero.) The remainder of the paper is devoted to proving several special cases of Conjecture 5.6.

Lemma 5.7. Suppose $\operatorname{Ran} T$ is dense in $\mathcal{H}$. Then for almost all $\mathbb{E} \in Z$, $\operatorname{Ran} T(\xi)$ is dense in $\mathcal{H}(\xi)$.

Proof. We will assume $\mathcal{H}_{0}$ is infinite dimensional and $\nu(Z)<\infty$; trivial modifications of the argument adapt the proof to the remaining cases. Let $\left\{e_{n}\right\}_{n=1}^{\infty}$ be an orthonormal basis for $\mathcal{H}_{0}$. Note that for almost all $\mathcal{E},\left\{T(\mathcal{G}) e_{n}\right\}_{n=1}^{\infty}$ is total in $\operatorname{Ran} T(\mathcal{E})$. For each $n$, let $x_{n}(\xi)$ be the projection of $e_{n}$ orthogonal to Ran $T(\mathcal{E})$; it is easily seen that the $\left\{x_{n}\right\}$ are measurable.

Define $y \in \mathcal{H}$ by

$$
y(\mathcal{E})= \begin{cases}x_{n}(\mathcal{E}) & \text { if } x_{1}(\mathcal{E})=\cdots x_{n-1}(\mathcal{E})=0 \\ & \text { but } x_{n}(\mathcal{E}) \neq 0 \\ 0 & \text { if } x_{n}(\mathcal{E})=0 \text { for all } n .\end{cases}
$$

Then $y \perp \operatorname{Ran} T$. Since $\operatorname{Ran} T$ is dense, $y=0$. Thus for almost all $\mathscr{E}, x_{n}(\mathscr{E})=0$ for all $n$. But then $\operatorname{Ran} T(\mathcal{E})$ is dense for almost all $\mathcal{E}$, and the proof is complete.

Theorem 5.8. Suppose $T(\mathcal{E})$ bas nontrivial null space for eacb $\mathcal{E} \in Z$. Then $T$ bas nontrivial null space and bence $T$ bas a byperinvariant subspace.

Proof. For each $\mathcal{E} \in Z, T^{*}(\mathcal{E})$ has nondense range. Thus $T^{*}$ has nondense range and $T$ has nontrivial null space. 
By virtue of Corollary 5.4, the conclusion of Theorem 5.8 remains valid under the (weaker) assumption that $T(\tilde{E})$ have 0 as an eigenvalue for each $\tilde{E}$ in some set of positive measure. Similar comments apply to the results below.

Theorem 5.9. Suppose $\sigma(T(\mathcal{E}))$ is disconnected for each $\mathcal{E} \in Z$. Then $T$ bas a byperinvariant subspace.

Proof. Applying Theorem 4.6, we find an appropriate set $E$ such that $T_{E}$ has disconnected spectrum. Thus $T_{E}$ (Proposition $5.1(6)$ ), and hence $T$, also have hyperinvariant subspaces.

Theorem 5.10. Suppose $T$ is nonscalar and $\sigma(T(\xi))$ consists entirely of point spectrum for eacb $\mathcal{E} \in Z$. Then $T$ bas a byperinvariant subspace.

Proof. If some fixed $\lambda$ belongs to $\sigma(T(\mathcal{E})$ ) for almost all $\mathcal{E}$, then $\lambda$ is an eigenvalue for $T$ and we are done by Proposition 5.1(1). Thus we may assume no $\lambda$ belongs to $\sigma(T(\xi))$ for almost all $\tilde{E}$.

Claim. For some square $R$ in $\mathrm{C},\left\{\mathcal{G}_{\mid} \sigma(T(\xi))\right.$ intersects $\left.R\right\}$ is proper. Indeed suppose not. Using the method of bisection, we find a sequence of squares $\left\{R_{n}\right\}$ satisfying:

(1) $R_{n} \subseteq R_{n-1}$;

(2) the sides of $R_{n}$ are half as long as those of $R_{n-1}$;

(3) $\mathrm{sp}^{-1}\left(\left\langle R_{n}\right\rangle\right)$ is a set of full measure in $Z$.

But then $\bigcap_{n=1}^{\infty} R_{n}$ is a single point $\lambda$ which would belong to $\sigma(T(\mathcal{G}))$ for almost

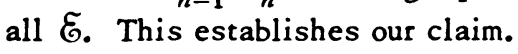

Pick sets $E_{1}$ and $E_{2}$ in $Z$ of finite positive measure such that sp(E) intersects $R$ for each $\mathcal{E} \in E_{1}$, but for no $\mathcal{E} \in E_{2}$. Replacing $E_{2}$ by a smaller set if necessary, we can even assume $\operatorname{dist}\left(R, \sigma\left(T_{E_{2}}\right)\right)$ positive.

Composing $\phi_{0}$ with sp, we get a measurable function $\lambda: E_{1} \rightarrow \mathbf{C}$ such that $\lambda(\mathcal{E}) \epsilon \sigma(T(\mathcal{E}))$ for each $\mathcal{E} \in E_{1}$. Moreover, for each $\mathscr{E} \in E_{1},(T(\mathcal{E})-\lambda(\mathcal{E}) I)$ has zero as an eigenvalue. Applying Theorem 5.8, we get a vector $x \in \mathcal{H}_{E_{1}}$ such that $T(\mathscr{G})_{x}(\mathcal{E})=\lambda(\mathscr{E})_{x}(\mathcal{E})$ for almost all $\mathscr{E}$ in $E_{1}$; we can also assume $\|x(\xi)\| \equiv 1$.

Set $\mathbb{M}=\left\{f(\cdot)_{x}(\cdot) \in \mathcal{H}_{E_{1}} \mid f \in L_{2}\left(E_{1}, \nu\right)\right\}$ and $\pi=\mathcal{H}_{E_{2}}$. Note that $\sigma\left(\left.T\right|_{\Re}\right)=$ ess ran $\lambda \subseteq R$ and $\sigma\left(\left.T\right|_{\Re}\right)=\sigma\left(T_{E_{2}}\right)$ is disjoint from $R$. Moreover $\mathbb{M}$ is invariant under $T$ and $\pi$ reduces $T$. Hence $T$ has a hyperinvariant subspace by Proposition 5.1(5).

Corollary 5.11. Suppose $T$ is nonscalar and $T(E)$ satisfies a (perbaps different) polynomial equation for each $\xi \in Z$. Then $T$ bas a byperinvariant subspace. 
Proof. Every point in $\sigma(T(\mathcal{E}))$ is an eigenvalue.

Corollary 5.12. Suppose $\mathcal{H}_{0}$ is finite dimensional. Then every nonscalar decomposable operator in $\mathcal{L}(\mathcal{H})$ bas a byperinvariant subspace.

Corollary 5.13 (Hoover). Every nonscalar n-normal operator bas a byperinvariant subspace.

Proofs of Hoover's theorem can be found in [0] and [13]. They depend on the fact that in expressing an $n$-normal operator as a direct integral, the measure space $(Z, \nu)$ can be taken to be perfect; since $\mathcal{H}_{0}$ is finite dimensional, this allows them to use "continuity arguments" in place of the "measuretheoretic arguments" of this paper. These methods do not seem to apply when $\mathcal{H}_{0}$ is infinite dimensional.

It is somewhat disconcerting that the hypothesis of Theorem 5.10 demands that $\sigma(A(\varepsilon))$ consist entirely of point spectrum. The final corollary indicates conditions under which this can be avoided.

Corollary 5.14. Suppose $T$ is not scalar and $\sigma(T(\mathcal{G}))$ bas at least one eigenvalue for each $\mathcal{E} \in Z$. Suppose moreover that either

(1) $\sigma(T(\mathscr{G}))$ is finite for each $\mathbb{E} \in Z$, or

(2) $T(\mathcal{E})$ is compact for each $\mathbb{E} \in Z$.

Then $T$ has a hyperinvariant subspace.

Proof. Set $E=\{\mathcal{E} \in Z \mid \sigma(T(\mathcal{E}))$ does not consist of a single point $\}$. $E$ is measurable since $\{K \in \mathcal{C} \mid K$ is a singleton $\}$ is closed in $\mathcal{C}$. If $E=\varnothing$, apply Theorem 5.10. Otherwise $T_{E}$ (and hence $T$ ) has a hyperinvariant subspace by Theorem 5.9.

Concluding remark. For ease of exposition, in our definition of direct integral, we assumed $\mathcal{H}(\mathcal{E})$ was the same Hilbert space for each $\mathcal{E}$. For the more general definitions, see $\S$ II.1 of [3].

As the reader can easily verify for himself, every theorem about decomposable operations stated in this paper is true in the more general case. This is a consequence of the following facts:

(1) Proposition 1(i) on p. 144 of [3],

(2) Theorem 2 on p. 167 of [3], and

(3) unitarily equivalent operators have the same spectrum.

Acknowledgment. Many of the results of this paper are adapted from the author's thesis written under the direction of Professor Carl Pearcy. The author would like to thank Professor Pearcy for his advice and encouragement. 


\section{REFERENCES}

1. T. R. Chow, A spectral theory for direct integrals of operators, Math. Ann. 188 (1970), 285-303. MR 42 \#3598.

2. D. Deckard and C. Pearcy, On matrices over the ring of continuous complex valued functions on a Stonian space, Proc. Amer. Math. Soc. 14 (1963), 322-328. MR 26 \#5438.

3. J. Dixmier, Les algèbres d'opérateurs dans l'espace Hilbertien, 2nd ed., Gauthier-Villars, Paris, 1969.

4. R. G. Douglas and C. Pearcy, Hyperinvariant subspaces and transitive algebras, Michigan Math. J. 19 (1972), 1-12. MR 45 \#4186.

5. F. Gilfeather, On a functional calculus for decomposable operators and applications to normal, operator-valued functions, Trans. Amer. Math. Soc. 176 (1973), 369-383.

6. - Operator-valued roots of abelian analytic functions, Bull. Amer. Math. Soc. 20 (1973), A154.

7. P. Halmos, A Hilbert space problem book, Van Nostrand, Princeton, N. J., 1967. MR 34 \#8178.

8. F. Hausdorff, Mengenlehre, 3rd. ed., de Gruyter, Berlin, 1937; English transl., Chelsea, New York, 1957. MR 19, 111.

9. T. Hoover, Hyperinvariant subspaces for n-normal operators, Acta. Sci. Math. (Szeged) 32 (1971), 449-461.

10. K. Kuratowski and C. Ryll-Nardzewski, A general theorem on selectors, Bull. Acad. Polon. Sci. Sér. Sci. Math. Astronom. Phys. 13 (1965), 397-403. (Russian) MR 32 \#6421.

11. E. Michael, Topologies on spaces of subsets, Trans. Amer. Math. Soc. 71 (1951), 152-182. MR 13, 54 .

12. C. M. Pearcy, Entire functions on infinite von Neumann algebras of type I, Michigan Math. J. 11 (1964), 1-7. MR 28 \#4381.

13. H. Radjavi and P. Rosenthal, Hyperinvariant subspaces for spectral and n-normal operators, Acta. Sci. Math. (Szeged) 32 (1971), 121-125.

DEPARTMENT OF MATHEMATICS, UNIVERSITY OF GEORGIA, ATHENS, GEORGIA 30602 